\title{
Heterostructure infrared photodiodes
}

\author{
Antoni Rogalski \\ Institute of Applied Physics, Military University of Technology, 00-908 Warsaw, Poland
}

\begin{abstract}
HgCdTe}$ remains the most important material for infrared photodetectors despite numerous attempts to replace it with alternative materials such as closely related mercury alloys (HgZnTe, HgMnTe), Schottky barriers on silicon, SiGe heterojunctions, AlGaAs multiple quantum wells, GaInSb strain layer superlattices, high temperature superconductors and especially two types of thermal detectors: pyroelectric detectors and silicon bolometers. It is interesting, however, that none of these competitors can compete in terms of fundamental properties. In addition, $\mathrm{HgCdTe}$ exhibits nearly constant lattice parameter that is of extreme importance for new devices based on complex heterostructures. The development of sophisticated controllable vapor phase epitaxial growth methods, such as MBE and MOCVD, has allowed fabrication almost ideally designed heterojunction photodiodes. Examples of novel devices based on heterostructures operating in long wavelength, middle wavelength and short wavelength spectral ranges are presented.
\end{abstract}

Keywords: $n$-on- $p$ HgCdTe photodiodes, InGaAs photodiodes, dual band-gap detectors

Paper received 25.08.99; revised manuscript received 01.12.99; accepted for publication 14.03.00.

\section{Introduction}

Recent success in applying infrared (IR) technology to remote sensing problems became possible due to the successful development of high-performance IR detectors over the last five decades. Many materials have been investigated in the IR field. Interest has centered mainly on the wavelengths of the two atmospheric windows $3-5 \mu \mathrm{m}$ and 8-14 $\mu \mathrm{m}$, though in recent years there has been increasing interest in longer wavelengths stimulated by space applications.

Recently, more interest has been focused on $p$ - $n$ junctions heterostructures $[1,2]$. Photodiodes with their very low power dissipation, easy multiplexing on focal plane silicon chip and less stringent noise requirements for the readout devices and circuits, can be assembled in twodimensional (2-D) arrays containing a very large $\left(\approx 10^{6}\right)$ number of elements, limited only by existing technologies. Systems based upon such focal plane arrays (FPAs) can be smaller, lighter, with lower power consumption, and can result in much higher performance than systems based on first generation detectors [3,4]. Photodiodes can also have less low frequency noise, faster response time, and the potential for a more uniform spatial response across each element. However, the more complex processes needed for photovoltaic detectors have influence on slower development and industrialization of the second generation systems, particularly for large arrays.

\section{Fundamental limitation to photodiode per- formance}

The photodetector is a slab of homogeneous semiconductor with the actual "electrical" area $A_{e}$ that is coupled to a beam of infrared radiation by its optical area $A_{0}$ (see Fig. 1). Usually, the optical and electrical areas of the device are the same or close. The use of optical concentrators can increase the $A_{0} / A_{e}$ ratio.

The current responsivity of the photodetector is determined by the quantum efficiency $\eta$ and by the photoelectric gain $g$. The quantum efficiency value describes how well the detector is coupled to the radiation to be detected. It is usually defined as the number of electronhole pairs generated per incident photon. The idea of photoconductive gain $g$ was put forth by Rose $\mathrm{e}^{5}$ as a simplifying concept for the understanding of photoconductive phenomena and is now widely used in the field. The photoelectric gain is the number of carriers passing contacts per one generated pair. This value shows how well the generated electron-hole pairs are used to generate the current response of a photodetector. Both values are assumed here as constant over the volume of the device.

The spectral current responsivity is equal to

$$
R_{i}=\frac{\lambda \eta}{h c} q g
$$




\section{A. Rogalski: Heterostructure infrared photodiodes}

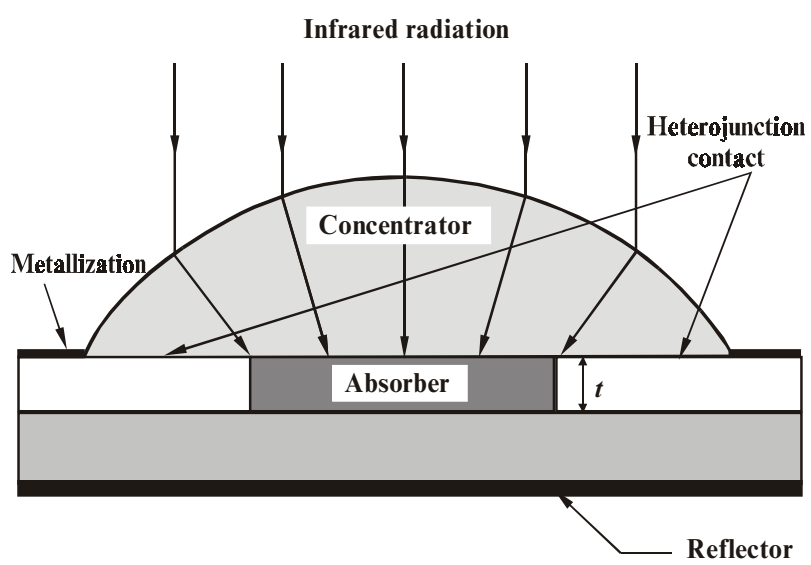

Fig. 1. Schematic of $3 D$ heterostructure photodetector (after Ref. 4).

where $\lambda$ is the wavelength, $h$ is the Planck's constant, $c$ is the light velocity, and $q$ is the electron charge. Assuming that the current gains for photocurrent and noise current are the same, the current noise due to generation and recombination processes is $[5,6]$

$$
I_{n}^{2}=2(G+R) A_{e} t \Delta f q^{2} g^{2}
$$

where $G$ and $R$ are the generation and recombination rates, $\Delta f$ is the frequency band, and $t$ is the thickness of the detector.

Detectivity $D^{*}$ is the main parameter characterizing normalized signal to noise performance of detectors and can be defined as

$$
D^{*}=\frac{R_{i}\left(A_{0} \Delta f\right)^{1 / 2}}{I_{n}}
$$

According to Eqs (1)-(3)

$$
D^{*}=\frac{\lambda}{h c}\left(\frac{A_{0}}{A_{e}}\right)^{1 / 2} \eta[2(G+R) t]^{-1 / 2}
$$

For a given wavelength and operating temperature, the highest performance can be obtained by maximizing $\eta /[t(G+R)]^{1 / 2}$. It means that high quantum efficiency must be obtained with a thin device. In further considerations we assume that $A_{0} / A_{e}=1$.

Assuming a single pass of the radiation and negligible frontside and backside reflection coefficients, the quantum efficiency and detectivity are

$$
\begin{aligned}
& \eta=1-\exp (-\alpha t) \\
& \left.D^{*}=\frac{\lambda}{h c}\left(1-e^{-\alpha t}\right) 2(G+R) t\right]^{-1 / 2}
\end{aligned}
$$

where $\alpha$ is the absorption coefficient.

The highest detectivity can be obtained for $t=1.26 / \alpha$ for which $\left(1-e^{\alpha t}\right) t^{-1 / 2}$ achieves a maximum value of
$0.62 \alpha^{1 / 2}$. This thickness is the best compromise between the requirements of high quantum efficiency and low thermal generation. In this optimum case $\eta=0.716$, and detectivity is equal

$$
D^{*}=0.45 \frac{\lambda}{h c}\left(\frac{\alpha}{G+R}\right)^{1 / 2}
$$

To achieve a high performance the thermal generation must be possibly suppressed to the lowest level. This is usually done with cryogenic cooling of the detector. For practical purposes, the ideal situation occurs when the thermal generation is reduced below the optical generation.

At equilibrium the generation and recombination rates are equal, and we have

$$
D^{*}=0.31 \frac{\lambda}{h c}\left(\frac{\alpha}{G}\right)^{1 / 2}
$$

The ratio of absorption coefficient to the thermal generation rate, $\alpha / G$, is the fundamental figure of merit of any material for infrared photodetectors which directly determines the detectivity limits of the devices [6]. An optimized photodetector of any type should provide the highest ratio of the optical-to-thermal generation rates. Any potential material should be compared on this basis.

IR photodetectors are traditionally related to photoconductive and photovoltaic detectors basing on the principle by which optically generated carriers are detected as a change in voltage or current across the element. The recent advances in heterostructure devices such as development of the heterojunction photoconductors, double layer heterostructure photodiode and introduction of nonequilibrium mode of operation make this distinction not so clear, however.

An optimized photodetector of any type should consist of the following regions (see Fig. 1) [4]:

- Lightly doped narrow gap semiconductor region, which acts as an absorber of IR radiation. Its band gap, doping and geometry should be selected.

- Electric contacts to the narrow gap region which sense optically generated charge carriers. Contacts should not contribute to the dark current of the device.

Passivation of the narrow gap region. The surfaces of the absorber regions must be insulated from the ambient by a material that also does not contribute to the generation of carriers. In addition, the carriers, which are optically generated in the absorber, are kept away from surfaces, where recombination can reduce the quantum efficiency. For the best sensitivity the frontside face should perfectly transmit IR radiation.

Refractive, reflective or diffractive concentrator of IR radiation.

- Backside mirror for double pass of IR radiation.

In practice, such a device can be obtained using 3-D gap and doping engineering, with the narrow gap absorber buried in a wide gap semiconductor. The undoped wide gap material can be used as a window, and/or a concentrator of 


\section{A. Rogalski: Heterostructure infrared photodiodes}

incoming radiation. Doped $n$ - and $p$-type semiconductors are used for contacts. Thermal generation in the contact regions is virtually eliminated by making them wide gap.

The generation-recombination processes are associated with the predominant recombination mechanisms. There are three important generation and recombination mechanisms: Shockley-Read, radiative, and Auger mechanisms. The Shockley-Read mechanism occurs via lattice defects and impurity energy levels within the forbidden energy gap. This mechanism set can be controlled by the procedure used to grow the material; consequently, the Shockley-Read process is not a fundamental limit to the performance of the detector. The radiative generation-recombination and Auger mechanisms are fundamental band-to-band processes, which are determined by the electronic band structure of the semiconductor. It appears that Auger mechanisms dominate generation and recombination processes in high-quality InSb-like narrow gap semiconductors. The generation rate due to the Auger 1 and Auger 7 processes can be described as [7, 8]

$$
G_{A}=\frac{n}{2 \tau_{A 1}^{i}}+\frac{p}{2 \tau_{A 7}^{i}}=\frac{1}{2 \tau_{A 1}^{i}}\left(n+\frac{p}{\gamma}\right),
$$

where $\tau_{A 1}^{i}$ and $\tau_{A 7}^{i}$ are the intrinsic Auger 1 and Auger 7 recombination times. Then Auger dominated detectivity is equal [see Eq. (4)]

$$
D^{*}=\frac{\lambda}{2^{1 / 2} h c} \frac{\eta}{t^{1 / 2}}\left(\frac{\tau_{A 1}^{i}}{n+p / \gamma}\right)^{1 / 2}
$$

where $\gamma=\tau_{A 7}^{i} / \tau_{A 1}^{i}$. Since the resulting Auger generation rate achieves its minimum for $p=\gamma^{1 / 2} n_{i}$, it leads to important conclusion about optimum doping. The optimum performance of Auger limited detectors can be achieved with a lightly $p$-type doping with whole concentration $p=\gamma^{1 / 2} n_{i}$.

Assuming that the saturation dark current $I_{s}$ of a photodiode is only due to thermal generation in the base layer and that its thickness is low compared to the diffusion length, we have $[7,8]$

$$
I_{S}=q G A t
$$

where $G$ is the generation rate in the base layer. Because the zero bias resistance-area product is

$$
R_{0} A=\frac{k T A}{q I_{s}}
$$

so

$$
R_{0} A=\frac{k T}{q^{2} G t}
$$

Taking into account the Auger 7 mechanism in extrinsic $p$-type region of $n^{+}$-on- $p$ photodiode, we receive

$$
R_{0} A=\frac{k T \tau_{A 7}^{i}}{q^{2} N_{a} t}
$$

and the same equation for $p$-on- $n$ photodiode

$$
R_{0} A=\frac{k T \tau_{A 1}^{i}}{q^{2} N_{d} t},
$$

where $N_{a}$ and $N_{d}$ are the acceptor and donor concentrations in the base region, respectively.

\section{HgCdTe ternary alloy for infrared detectors}

During the past four decades mercury cadmium telluride ( $\mathrm{HgCdTe}$ ) has become the most important semiconductor for the middle and long wavelength $(\lambda=3-30 \mu \mathrm{m})$ infrared (IR) photodetectors [1, 2, 5, 9-13]. The short wavelength region has been dominated by III-V compounds (InGaAs, InAsSb, InGaSb).

There have been numerous attempts to replace $\mathrm{HgCdTe}$ with alternative materials. At present, several other variable gap alloy systems are known including closely related mercury alloys $\mathrm{HgZnTe}, \mathrm{HgMnTe}$, lead tin tellurides and selenides, InAsSb, III-VI compounds with thallium and bismuth, free-carrier detectors and low dimensional solids [14-17].

The main motivations, besides the numerous attempts to replace $\mathrm{HgCdTe}$, are technological problems of this material. One of them is a weak $\mathrm{Hg}$-Te bond, which results in bulk and surface and interface instabilities. Uniformity and yield are still issues. Nevertheless, $\mathrm{HgCdTe}$ remains the leading semiconductor for IR detectors. The most important reasons for this are:

Not any of the new materials offers fundamental advantages over $\mathrm{HgCdTe}$. While the figure of merit, $(\alpha / G)^{1 / 2}$, of various narrow gap semiconductors seems to be very close to that of $\mathrm{HgCdTe}$, the free carrier detectors and GaAs/AlGaAs superlattice devices have several order of magnitude smaller $\alpha / G$.

- $\mathrm{HgCdTe}$ exhibits extreme flexibility, it can be tailored for optimized detection at any region of IR spectrum, dual and multicolor devices can be easily constructed.

The present development of IR photodetectors has been dominated by complex band gap heterostructures. Among various variable band gap semiconductor alloys, $\mathrm{HgCdTe}$ is the only one material covering the whole IR spectral range having nearly the same lattice parameter. The difference of lattice parameter between $\mathrm{CdTe}\left(E_{g}=\right.$ $1.5 \mathrm{eV})$ and $\mathrm{Hg}_{0.8} \mathrm{Cd}_{0.2} \mathrm{Te}\left(E_{g}=0.1 \mathrm{eV}\right)$ is $\approx 0.2 \%$. Replacing small fraction of $\mathrm{Cd}$ with $\mathrm{Zn}$ or Te with $\mathrm{Se}$ can compensate the residual lattice mismatch. The independence of lattice parameter on composition is a major advantage of $\mathrm{HgCdTe}$ over any other materials.

Actually, heterostructures do not offer any adherent fundamental advantages over homostructures for the conventional equilibrium mode devices. The fundamental limits to performance of IR detectors are imposed by una- 


\section{A. Rogalski: Heterostructure infrared photodiodes}

voidable physics of optical and thermal generation in the narrow gap base region of a photodetector. Nevertheless, heterojunctions are helpful in achieving high performance in practice. For example, the narrow gap $\mathrm{HgCdTe}$ which absorb infrared radiation can be buried encapsulated in wider gap $\mathrm{HgCdTe}$ preventing instabilities due to the weak $\mathrm{Hg}$-Te bonds. In addition, heterostructures can be used for non-equilibrium device which potential performance can be much higher than that of the conventional ones [12].

Epitaxy is the preferable technique to obtain devicequality $\mathrm{HgCdTe}$ epilayers for IR devices. The epitaxial techniques offer, in comparison with bulk growth techniques, the possibility to grow large area epilayers and sophisticated layered structures with abrupt and complex composition and doping profiles, which can be configured to improve the performance of photodetectors. The growth is performed at low temperatures, which makes it possible to reduce the native defects density. Due to the low mercury pressures there is no need for thick-walled ampoules and the growth can be carried out in reusable production-type growth systems. The as-grown epilayers can be low-temperature annealed in situ.

Epitaxial growth of the $\mathrm{HgCdTe}$ detector array on a Si substrate, rather than CdZnTe, has emerged as a particularly promising approach to scale up wafer dimensions and achieve a cost-effective number of array die from each processed wafer. In addition to the potential for increasing wafer size from the current $30 \mathrm{~cm}^{2}$ for $\mathrm{CdZnTe}$ substrates to $125 \mathrm{~cm}^{2}$ for Si substrates, the growth of $\mathrm{HgCdTe}$ FPAs on Si substrates offers other compelling advantages such as creation of a thermal-expansion matched hybrid structure, superior substrate mechanical strength and flatness, elimination of impurity out diffusion from the substrate, and compatibility with automated wafer processing and handling methodologies.

Among various epitaxial techniques, the liquid phase epitaxy (LPE) is the most matured method enabling growth of device-quality homogeneous layers and multilayered structures. LPE growth must be performed at relatively high growth temperature with adherent interdiffusion and resulting graded interfaces. The recent efforts are mostly on low growth temperature techniques; metalorganic chemical vapor deposition (MOCVD) and molecular beam epitaxy (MBE). MOCVD is a non-equilibrium method, which appears to be the most promising for future large-scale and low-cost production of epilayers. The important advantage of this method is reduced growth temperature and the ability to modify the growth conditions during growth to obtain required band gap and doping profiles.

Intensive studies are currently under way on MBE. This technique offers unique capabilities in material and device engineering including the lowest growth temperature, superlattice growth and potential for the most sophisticated composition and doping profiles. The growth temperature is less than $200^{\circ} \mathrm{C}$ for $\mathrm{MBE}$ but around $350^{\circ} \mathrm{C}$ for MOCVD, making it more difficult to control the $p$-type doping in the MOCVD due to the formation of $\mathrm{Hg}$ vacan- cies at higher growth temperatures. The main drawback of both technologies is a high cost of equipment and maintenance. This prevents wider spread of the methods.

\section{Double layer heterojunction photodiodes}

The realization of $\mathrm{HgCdTe}$ heterostructure photodiodes has based on $\underline{p}^{+}$-on- $n$ structure (symbol " + " denotes strong doping, underlined "_" wider gap). In such diodes, the lightly doped narrow gap absorbing region ("base" of the photodiode), determines the dark current and photocurrent. In these photodiodes the base $n$-type layers are sandwiched between CdZnTe substrate and highdoped, wider-gap regions. Due to backside illumination (through CdZnTe substrate) and internal electric fields (which are "blocking" for minority carriers), influence of surface recombinations on the photodiodes performance is eliminated. The influence of surface recombination can be also prevented by the use of suitable passivation. Both optical and thermal generations are suppressed in the $\underline{p}^{+}$-region due to wide gap. Thus $R_{0} A$ product of double-layer heterojunction (DLHJ) structure is higher than homostructure.

The thickness of the base region should be optimized for near unity quantum efficiency and a low dark current. This is achieved with a base thickness slightly higher than the inverse absorption coefficient for single pass devices: $t=1 / \alpha$ (which is $\approx 10 \mu \mathrm{m}$ ) or half of the $1 / \alpha$ for double pass devices (devices supplied with a retroreflector). Low doping is beneficial for a low thermal generation and high quantum efficiency. Since the diffusion length in absorbing region is typically longer than its thickness, any carriers generated in the base region can be collected giving rise to the photocurrent.

The baseline detector architectures for short wavelength infrared (SWIR) InGaAs and $\mathrm{HgCdTe}$ DLHJ photodiodes are shown in Fig. 2. In the case of $\mathrm{HgCdTe}$ photodiodes, $n$-type base absorbing region is deliberately doped with indium at a level of about $(1-3) \times 10^{15} \mathrm{~cm}^{-3}$. $p$ - $n$ junction is formed using arsenic as the dopant at a level of about $10^{18} \mathrm{~cm}^{-3}$. To active As an acceptor, it must occupy a Te site in the lattice. Full As activation is achieved for annealing temperature of $300^{\circ} \mathrm{C}$ or higher. The junction are also formatted by As selective implantation through windows made on a mask of photoresist/ZnS and then diffusing the arsenic through the cap layer into the narrow-gap base layer. After implantation, the sample underwent two consecutive annealing, one at about $430^{\circ} \mathrm{C}$ (to diffuse the arsenic into the base layer) for approximately $10 \mathrm{~min}$. and the other at $250^{\circ} \mathrm{C}$ (to annihilate $\mathrm{Hg}$ vacancies formed in the $\mathrm{HgCdTe}$ lattice during growth and diffusion of arsenic) [18]. The electrical junction is positioned near the metallurgical interface and it is wise to place the junction in the small band gap layer to avoid deleterious effects on the quantum efficiency and dark currents. At present, most laboratories are using CdTe or CdZnTe (deposited by MBE, MOCVD, sputtering and ebeam evaporation) for photodiode passivation [18]. 




(b)

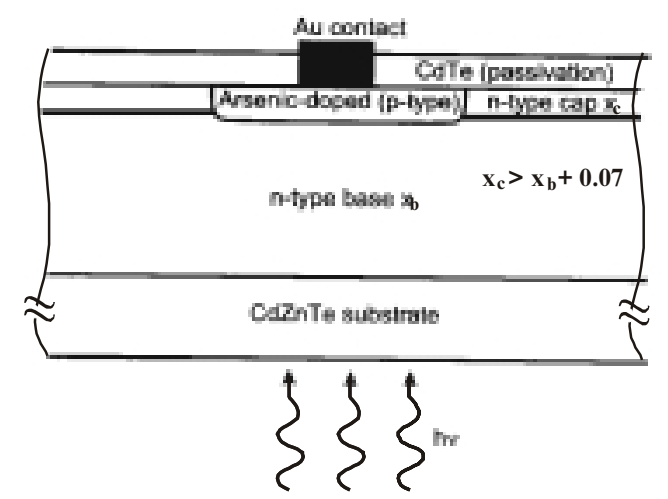

Fig. 2. Double layer planar heterostructure cross section for InGaAs (a) and HgCdTe (b) photodiodes.

\subsection{Long wavelength infrared photodiodes}

The dependence of the base region diffusion limited $R_{0} A$ product on the long wavelength cutoff for $p^{+}$-on- $n$ long wavelength IR (LWIR) $\mathrm{HgCdTe}$ photodiodes at different temperatures is shown in Fig. 3. This figure also includes the experimental data reported by many authors for DLHJ $p$-on- $n$ structures. The wider bandgap cap layer contributes a negligible amount of thermally generated diffusion current compared with that from an $n$-type absorber layer at $77 \mathrm{~K}$, and the upper experimental data are situated about a half of an order below ultimate theoretical predictions. With a lowering of the operation temperature of photodiodes, the discrepancy between the theoretical curves and experimental data increases, which is due to additional currents in the junctions (such as tunneling current or surface leakage current) that are not considered. Photodiodes with lower performance usually contain metallurgical defects such as dislocation clusters and loops, pin holes, striations, Te inclusions, and heavy terracing. Diodes with the highest performance at $40 \mathrm{~K}$ contain no visible defects ( $\mathrm{Hg}$ interstitials and vacancies). It should be noticed that the upper experimental data in very long wavelength range (above $14 \mu \mathrm{m}$ ) at lower temperatures $(40 \mathrm{~K})$ coincides very well with theoretical predictions. The best devices continued to be diffusion-current limited by Auger mechanism at zero bias to $35 \mathrm{~K}$. At $40 \mathrm{~K}$, the measured $R_{0} A$ is $2 \times 10^{4} \Omega \mathrm{cm}^{2}$ and the measured cutoff wavelength is $17.6 \mu \mathrm{m}$. At $35 \mathrm{~K}, R_{0} A$ is $2 \times 10^{5} \Omega \mathrm{cm}^{2}$ at cutoff wavelength $18.1 \mu \mathrm{m}$. Also the performance of photodiodes with cutoff wavelengths of $20.3 \mu \mathrm{m}$ at $40 \mathrm{~K}$ is diffusion limited and $R_{0} A$ products for the diodes reach values in the $10^{3} \Omega \mathrm{cm}^{2}$ range. There are the highest reported values at these long cutoffs wavelengths for any $\mathrm{HgCdTe}$ device.

Typical results achieved for LPE based $p$-on- $n$ DLHJ photodiodes include the following [18]:
- $R_{0} A$ values at $78 \mathrm{~K}$ which fit a diffusion-limited mechanism over a cutoff wavelength range from 9 to $11.5 \mu \mathrm{m}$ for material doped $n$ type at $2 \times 10^{15} \mathrm{~cm}^{3}$ and with lifetime $\approx 500 \mathrm{~ns}$,



Fig. 3. Dependence of the $R_{0} A$ product on the long wavelength cutoff for LWIR $\underline{p}^{+}-n \mathrm{HgCdTe}$ photodiodes at temperatures $\leq 77$ $\mathrm{K}$. The solid lines are calculated assuming that the performance of photodiodes are due to thermal generation governed by the Auger mechanism in the base $n$-type region of photodiodes with $t=10 \mu \mathrm{m}$ and $N_{d}=5 \times 10^{14} \mathrm{~cm}^{-3}$. The experimental values are taken from different papers marked inside the figure. 


\section{A. Rogalski: Heterostructure infrared photodiodes}

- when normalized to a cutoff wavelength of $10.5 \mu \mathrm{m}$, the average $R_{0} A$ produced under a background photon flux of $\approx 2 \times 10^{12}$ photons $/ \mathrm{cm}^{2}$ s is $300 \Omega \mathrm{cm}^{2}$ with a breakdown voltage of $600 \mathrm{mV}$,

- with a $f / 2$ cold shield to establish $\Phi_{B} \approx 3 \times 10^{16}$ photons $/ \mathrm{cm}^{2}$ s average $R_{0} A \approx 200 \Omega \mathrm{cm}^{2}$,

- at $40 \mathrm{~K}, R_{0} A$ is typically above the $6 \times 10^{5} \Omega \mathrm{cm}^{2}$ required for background limited performance at $\Phi_{B} \approx$ $1 \times 10^{12}$ photons $/ \mathrm{cm}^{2} \mathrm{~s}$,

- even the smallest diodes are not surface limited,

- these devices also withstand prolonged baking at $150^{\circ} \mathrm{C}$,

exceptionally low $1 / f$ noise (knee frequency $<0.1 \mathrm{~Hz}$ at $50 \mathrm{mV}$ reverse bias) has been observed.

\subsection{Middle wavelength infrared photodiodes}

Middle wavelength infrared (MWIR) HgCdTe photo-diodes were the first to be developed, and many mature technologies have been used to demonstrate FPAs [30, 31]. Since 1989, SBRC has successfully utilized "infinite-melt" vertical LPE technology from $\mathrm{Hg}$-rich solution to grow high-quality epitaxial $\mathrm{HgCdTe}$ on the Si-based alternative substrates for the fabrication of $p$-on- $n$ DLHJ detectors for high-performance MWIR FPAs. Tung et al. [32] reported large, up to $480 \times 640$, MWIR FPAs, grown on Si-based alternative substrates.

The MBE-grown devices on Si and CdZnTe appear to be identical [33]. Figure 4 presents a comprehensive comparison of the performance of MWIR $p^{+}$-on- $n \mathrm{HgCdTe}$ photodiodes on CdZnTe and Si substrates for cutoff wavelengths ranging from $3.5 \mu \mathrm{m}$ to $5 \mu \mathrm{m}$. The various data points are median values for mini-arrays included in test structures for each processed wafer. The devices with highest performance are processed from MBE-grown epilayers on bulk CdZnTe substrates. The shorter cutoff devices (with $\lambda_{c} \approx 3 \mu \mathrm{m}$ ) are diffusion-limited down to at least 125 $\mathrm{K}$. The devices with longer cutoff wavelength (with $\lambda_{c} \approx 5$ $\mu \mathrm{m})$ appear to be diffusion-limited down to approximately $110 \mathrm{~K}$. Below this temperature the experimental data obscure the probable onset of generation-recombination and/ or tunneling current limitations.

\subsection{Short wavelength infrared photodiodes}

Considerable progress in HgCdTe SWIR hybrid FPAs has been achieved in the last decade. At the beginning the detector arrays were fabricated using a $n^{+}$boron implanted process on $p$-type $\mathrm{HgCdTe}$ layers grown by LPE on CdTe or CdZnTe substrates [30]. Next, the PACE-1 process was adopted to fabricate large, $2.5-\mu \mathrm{m} 1024 \times 1024$ FPAs (HAWAII) for IR astronomy [34, 35]. Recently, DLPH $p$-on- $n$ photodiodes in molecular beam epitaxy (MBE) $\mathrm{HgCdTe}$ on CdZnTe substrates have been elaborated by As-ion implantation and the $p$-dopant activation by an open-tube $\mathrm{Hg}$ anneal [36].

Due to similar band structure of InGaAs and $\mathrm{HgCdTe}$ ternary alloys, the ultimate fundamental performance of

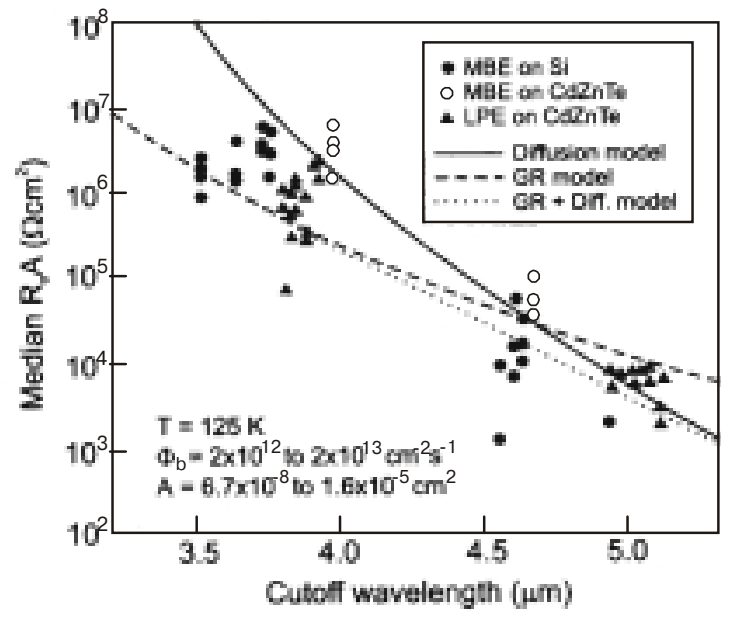

Fig. 4. Comparison of $125 \mathrm{~K}$ detector performance for MWIR $\mathrm{HgCdTe}$ photodiodes grown on $\mathrm{Si}$ and $\mathrm{CdZnTe}$ by MBE and photodiodes grown on $\mathrm{CdZnTe}$ by LPE. Each data point represents an array-median $R_{0} A$ product measured at $125 \mathrm{~K}$ (after Ref. 33).

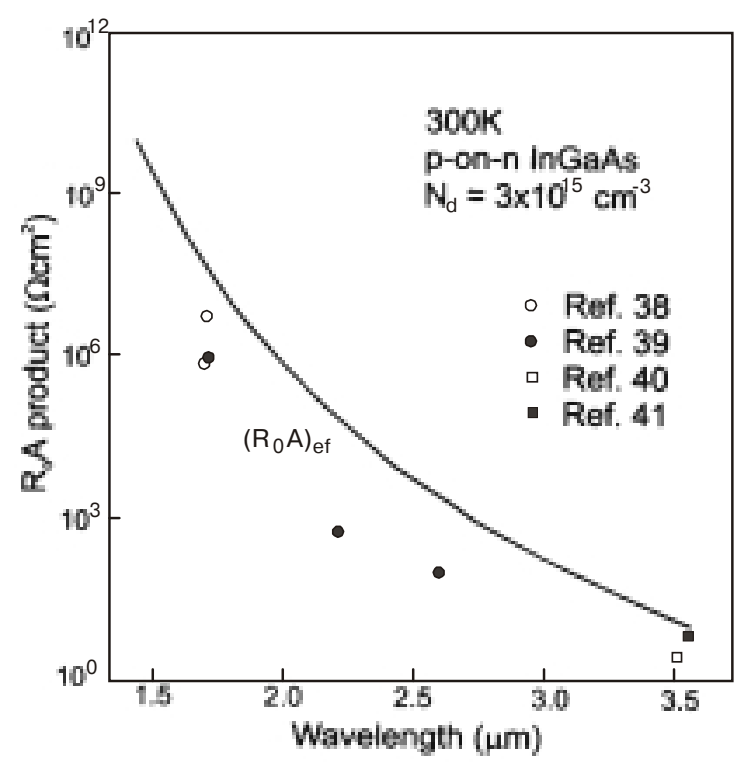

Fig. 5. The dependence of effective $R_{0} A$ product on the long wavelength cutoff for SWIR InGaAs photodiodes at room temperature. The calculations are performed assuming that the performance of photodiodes is due to fundamental generation-recombination processes in the base $n$-type region of photodiodes with $t=5 \mu \mathrm{m}$ and $N_{a}=3 \times 10^{15} \mathrm{~cm}^{-3}$. The experimental values are taken from Ref. 38 (०), Ref. 39 (•), 40 (ロ), and Ref. 41 (•) (after Ref. 37). 


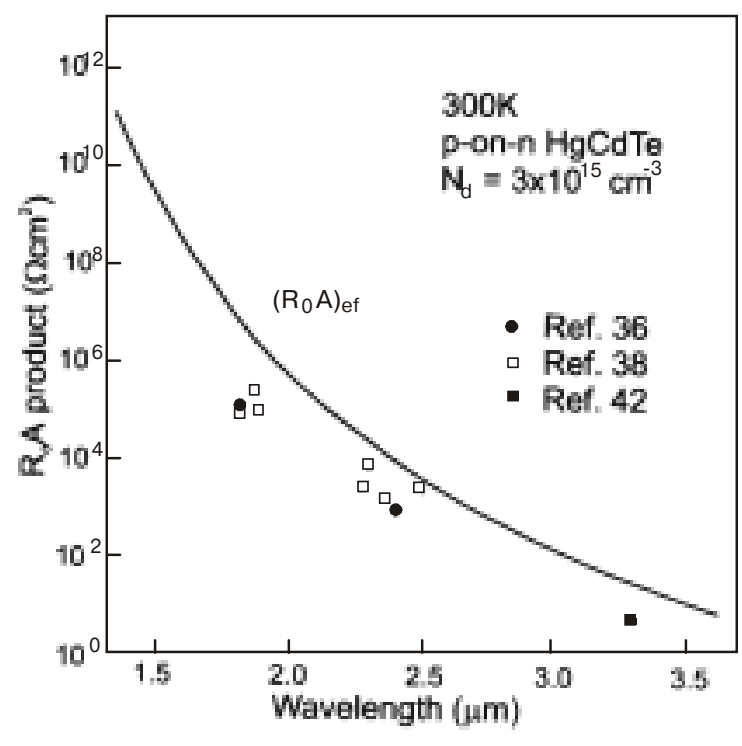

Fig. 6. The dependence of effective $R_{0} A$ product on the long wavelength cutoff for SWIR $\mathrm{HgCdTe}$ photodiodes at room temperature. The calculations are performed assuming that the performance of photodiodes is due to fundamental generation-recombination processes in the base $n$-type region of photodiodes with $t=5 \mu \mathrm{m}$ and $N_{a}=3 \times 10^{15} \mathrm{~cm}^{-3}$. The experimental values are taken from Ref. $36(\bullet)$, Ref. $38(\square)$, and Ref. 42 (•) (after Ref. 37).

both type of photodiodes are similar in the wavelength range $1.5<\lambda<3.7 \mu \mathrm{m}$ [37]. Next two figures (Figs 5 and 6) compare the ultimate performance of $n$-type base InGaAs and $\mathrm{HgCdTe}$ photodiodes with attainable experimental data. InGaAs photodiodes have shown high device performance close to theoretical limits for material whose composition is nearly matched to that of $\operatorname{InP}(\cong 1.7 \mu \mathrm{m}$ cut-off wavelength) and InAs ( $\cong 3.6 \mu \mathrm{m}$ cut-off wavelength). However, their performance decreases rapidly at intermediate wavelengths due to mismatch-induced defects with the substrate. On the other hand, SWIR $\mathrm{HgCdTe}$ photodiodes have good performance over a wider range of wavelengths. It is due to lattice match of active base photodiode layers with $\mathrm{CdZnTe}$ substrate and consequently lower influence of induced defects at interface on photodiode leakage current.

\section{Dual-band detectors}

As the IR technology continues to advance, there is a growing demand for multispectral detectors for advanced IR systems with better target discrimination and identification. Devices for sequential and simultaneous detection of two closely spaced sub-bands in the MWIR, sequential detection of MWIR and LWIR radiation as well as those for the sequential detection of two sub-bands in the LWIR have been demonstrated [43-50]. One of such two-color $\mathrm{HgCdTe}$ detectors that has been recently reported is the bias-selectable back-to-back photodiode [43-47]. This device is formed by sequentially growing $n$ $\underline{p}-\underline{n}$ structure with two HgCdTe photodiodes. The devices were grown on commercially available (211)B CdZnTe substrates (see Fig. 7). Critical step in device formation is connected with in situ doped 4- $\mu$ m thick $p$-type Asdoped MWIR layer with good structural and electrical properties. $n$-type conductivity in $\mathrm{HgCdTe}$ was obtained by doping the material with a group II or group VII impurity (usually indium). The top and bottom layers are the LW and MW absorbers with the middle layer being transparent. In one mode, the MW junction is reversebiased, producing a signal for the MWIR; and the LW junction is forward-biased, producing no signal. Reversing the bias polarity activates the LW junction but not the

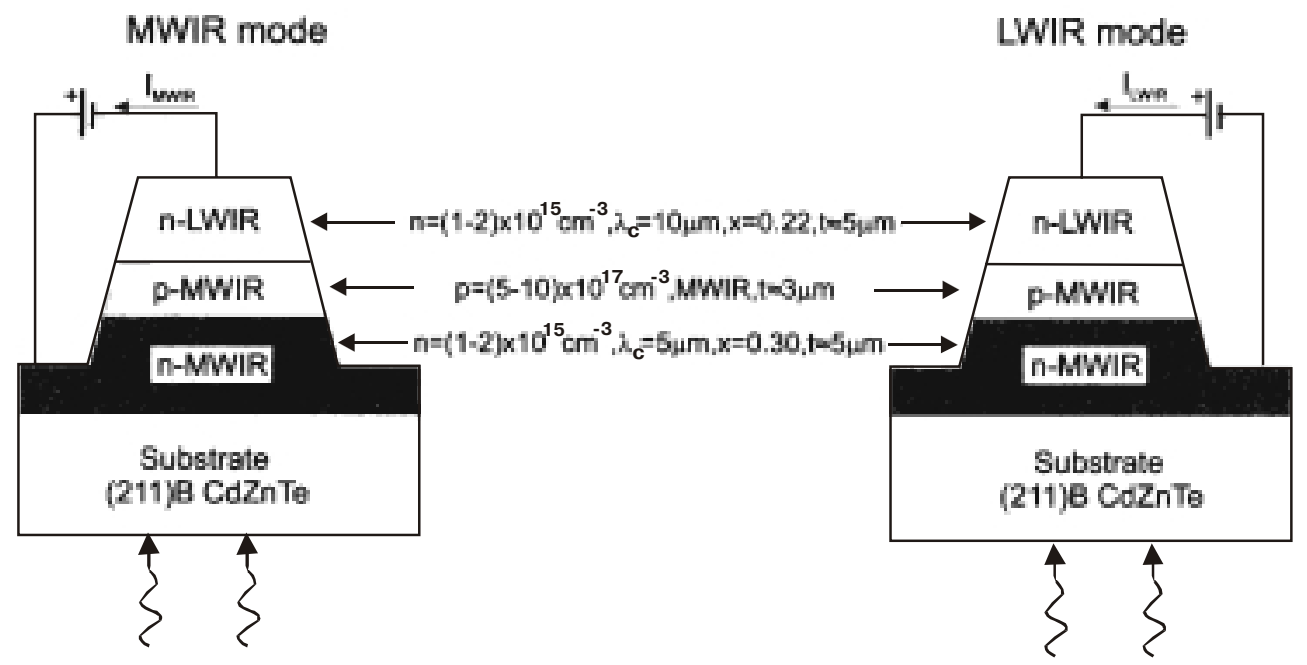

Fig. 7. MWIR and LWIR modes of operation of a sequential $n$ - $p$ - $n$ two-color detector achieved by switching the bias (after Ref. 46). 


\section{A. Rogalski: Heterostructure infrared photodiodes}

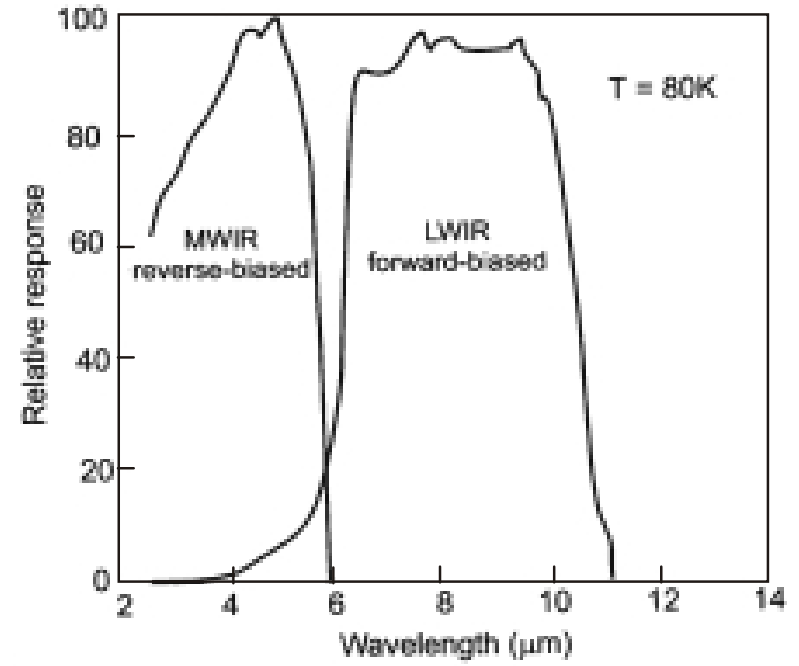

Fig. 8. Relative spectral response versus wavelength for dualband (MWIR and LWIR) detectors operating at reverse and forward-bias modes (after Ref. 46).

MW junction. The relative spectral response of this kind of detector is shown in Fig 8. The LW detectors were fabricated with $R_{0} A>100 \Omega \mathrm{cm}^{2}\left(\lambda_{c}=10.5 \mu \mathrm{m}\right)$ and the MW detectors with $R_{0} A>10^{5} \Omega \mathrm{cm}^{2}\left(\lambda_{c}=5.7 \mu \mathrm{m}\right)$ at $78 \mathrm{~K}$.

The problems with the bias selectable device are the following: its construction does not allow independent selection of the optimum bias voltage for each photodiode, and there can be substantial medium wavelength (MW) crosstalk in the long wavelength ( $\mathrm{LW}$ ) detector. Reine et al. $[45,48,49]$ have developed a new two-color $\mathrm{HgCdTe}$ IR detector which overcomes the problems of the bias- selectable device. Figure 9 shows cross section of the independently accessed back-to-back photodiode dual-band detector. The LW photodiode is a $p$-on- $n$ heterojunction, grown directly on top of the MW photodiode, which is an $n$-on- $p$ heterojunction. A thin $n$-type compositional barrier layer is placed between the $\mathrm{MW}$ and $\mathrm{LW}$ absorber layers. This barrier layer forms isotype $n-\underline{n}$ heterojunction at the interface, which prevents MW photocarriers from diffusing into the LW absorber layer and prevents LW photocarriers from diffusing into the MW absorber layer.

The above detectors with two bumps per unit cell for electrical, mechanical and thermal interconnections can be implemented in large dual-band IR hybrid FPAs. One bump contacts only the $p$-type region of the LW photodiode; the other bump contacts the $n$-type region of the LW photodiode, and therefore also the $n$-type region of the MW photodiode, through an over-the-edge metalization. This way the LW photodiode is accessed directly through the two bumps, while the MW photodiode is accessed through the contact to the $n$-type region and the array ground. This dual-band device showed that the MW $\underline{n}$-on- $\underline{p}$ homojunction photodiodes had good $R_{0} A$ products but the quantum efficiencies were anomalously low and in some cases the spectral responses were sharply peaked. To alleviate these problems, in situ growth of MW diodes in the $\underline{n}$-on- $\underline{p}$ heterojunction configuration by MOCVD has been carried out [48,49] (see structure shown in Fig. 9). In the $\underline{n}$-on- $p$ heterojunction, the absorber layer is the $n$-type layer as compared to the $p$-type layer in the $\underline{n}$-on- $\underline{p}$ homojunction, and significantly improved quantum efficiency of $65-80 \%$ (without anti-reflection coating) and classical spectral response have been obtained.

The experimentally demonstrated $64 \times 64$ dual-band array has a unit cell size of $75 \times 75 \mu \mathrm{m}^{2}$. The deep MW mesa etch was performed by ECR dry etching. The arrays
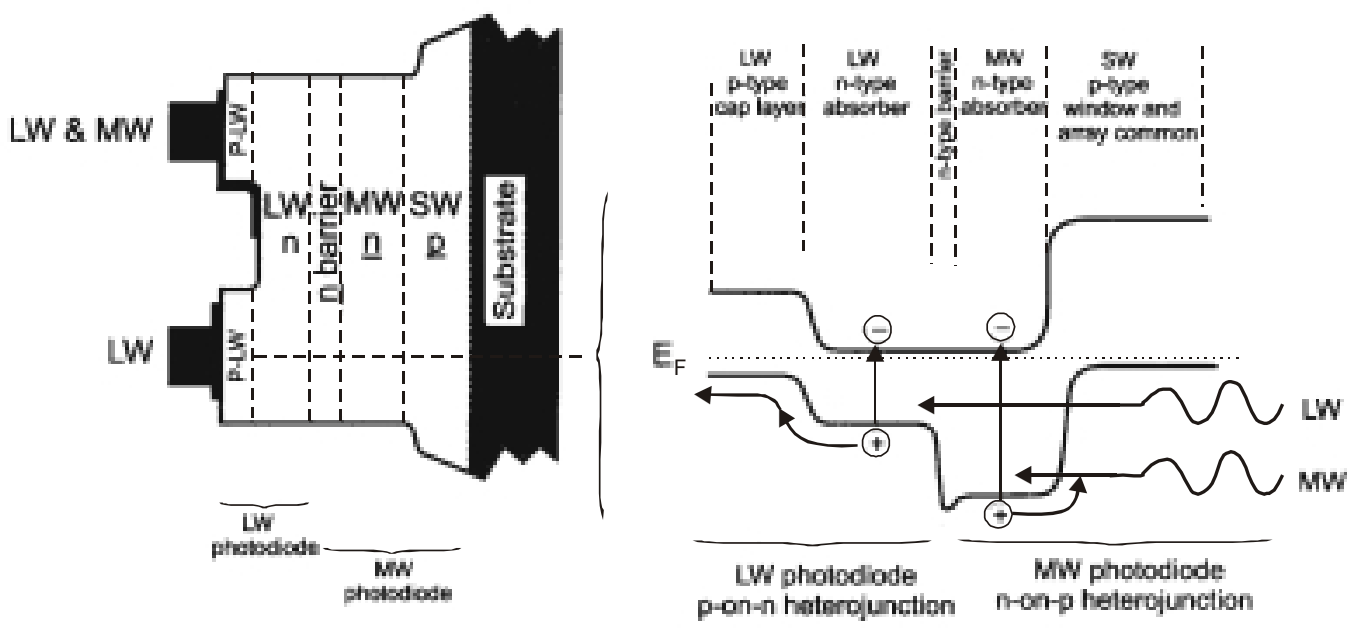

Fig. 9. Cross section and energy band profile of the independently accessed back-to-back HgCdTe photodiode dual-band detector (after Ref. 49). 


\section{A. Rogalski: Heterostructure infrared photodiodes}

were hybridized to a dual-band silicon multiplexer readout chip that allowed the MW and LW photocurrents to be integrated simultaneously and independently. In the FPA, the wide gap SW p-type layer is the common contact to all detectors in the array. The MW and LW average cutoff wavelengths at $77 \mathrm{~K}$ are in the 4.27-4.35 $\mu \mathrm{m}$ and 10.1$10.5 \mu \mathrm{m}$ ranges respectively. These staring dual-band FPAs exhibit high average quantum efficiency (MW: 79\%; LW: $67 \%$ ), high median detectivities (MW: $4.8 \times 10^{11}$ $\mathrm{cmHz}^{1 / 2} \mathrm{~W}^{-1}$; LW: $\left.7.1 \times 10^{10} \mathrm{cmHz}^{1 / 2} \mathrm{~W}^{-1}\right)$, and low median NETDs (MW: $20 \mathrm{mK}$; LW: $7.5 \mathrm{mK}$ for $T_{\mathrm{SCENE}}=295 \mathrm{~K}$ and $f / 2.9$ ).

A successful demonstration of the prototype $128 \times 128$ array operated as back-to-back diodes for the simultaneous detection of two closely spaced sub-bands in the MWIR spectrum has been described by Rajavel et al [50]. The device structures were delineated as mesa isolated structures and contacts were made to the top $n$-type layer and the intermediate $p$-type layer. Fill factors as high as $80 \%$ were achieved by using a single mesa structure to accommodate the two indium bump contacts required for each unit cell. The devices were characterized by $R_{0} A$ product in excess of $5 \times 10^{5} \Omega \mathrm{cm}^{2}$ at $78 \mathrm{~K}$, at $f / 2 \mathrm{FOV}$ and quantum efficiency greater than $70 \%$ in each band. Imagery was acquired at temperature as high as $180 \mathrm{~K}$ without any visible degradation in image quality.

\section{Conclusions}

To summarize, despite serious competition from alternative technologies and slower progress than expected, $\mathrm{HgCdTe}$ is unlikely to be seriously challenged for highperformance applications, applications requiring multispectral capability and fast response. The recent successes of competing cryogenically cooled detectors are due to technological, not fundamental issues. There are good reasons to think that the steady progress in epitaxial technology would make $\mathrm{HgCdTe}$ devices much more affordable in the near future. The much higher operation temperature of $\mathrm{HgCdTe}$ compared to Schottky barrier devices and low-dimensional solid devices may become a decisive argument in this case. The development of sophisticated controllable vapor phase epitaxial growth methods, such as MBE and MOCVD, has allowed to fabricate almost ideally designed heterojunction photodiodes. Such devices can be obtained using 3-D gap and doping engineering, with the narrow gap absorber buried in a wide gap semiconductor. The undoped wide gap material can be used as a window, and/or a concentrator of incoming radiation. Doped $n$ - and $p$-type semiconductors are used for contacts. Thermal generation in the contact regions is virtually eliminated by making them wide gap.

In applications for short-range thermal imaging systems a serious challenge comes from solid state arrays of thermal detectors (pyroelectric and bolometers) which are expected to take over and increase the market for uncooled short-range imaging systems.

The long term picture could change as a result of current research activity, both for single element detectors and arrays. Currently, no known variable gap material can offer fundamental advantages in terms of performance or cost of production. A challenge may come rather from materials exhibiting higher stability.

\section{Acknowledgement}

This work was partially supported by the KBN (Poland) under grant number PBZ 28.11/P6.

\section{References}

1. A. Rogalski, Infrared photovoltaic detectors // Opto-Electr. Rev. 5, pp. 205-216 (1997).

2. A. Rogalski and M. Razeghi, Narrow gap semiconductor photodiodes // Proc. SPIE 3287, pp. 2-13 (1998).

3. D. A. Scribner, M. R. Kruer and J. M. Killiany // Infrared focal plane array technology, Proc. IEEE 79, pp. 66-85 (1991).

4. J. Piotrowski and A. Rogalski, New generation of infrared photodetectors // Sensors and Actuators A 67, pp. 146-152 (1998).

5. A. Rose, Concepts in Photoconductivity and Allied Problems, Interscience, New York (1963).

6. J. Piotrowski and W. Gawron, Ultimate performance of infrared photodetectors and figure of merit of detector material // Infrared Phys. Technol. 38, pp. 63-68 (1997).

7. J. Piotrowski, $\mathrm{Hg}_{1-\mathrm{x}} \mathrm{Cd}_{\mathrm{x}} \mathrm{Te}$ detectors // in Infrared Photon Detectors, pp. 391-493, edited by A. Rogalski, SPIE Optical Engineering Press, Bellingham, (1995).

8. A. Rogalski and R. Ciupa, Long wavelength $\mathrm{HgCdTe}$ photodiodes: $n^{+}$-on- $p$ versus $p$-on- $n$ structures // J. Appl. Phys. 77, pp. 3505-3512 (1995).

9. A. Rogalski and J. Piotrowski, Intrinsic infrared detectors // Prog. Quant. Electr. 12, pp. 87-289 (1988).

10. J. Piotrowski, W. Galus, and M. Grudzien, Near room-temperature IR photo-detectors // Infrared Phys. 31, pp. 1-48 (1991).

11. C. T. Elliott and N. T. Gordon, Infrared detectors // in Handbook on Semiconductors, Vol. 4, pp. 841-936, edited by C. Hilsum, North-Holland, Amsterdam (1993).

12. J. Piotrowski, W. Gawron, and Z. Djuric, New generation of near-room-temperature photodetectors // Opt. Eng. 33, pp. 1413-1421 (1994).

13. K. Adamiec, A. Rogalski, and J. Rutkowski, Progress in infrared detector technology // J. Tech. Phys. 38, pp. 431488 (1997).

14. A. Rogalski, New Ternary Alloy Systems for Infrared Detectors, SPIE Optical Engineering Press, Bellingham (1994).

15. Infrared Photon Detectors, edited by A. Rogalski, SPIE Optical Engineering Press, Bellingham (1995).

16. J.L. Lee, J.D. Kim, and M. Razeghi, Novel InTlSb alloy for uncooled long-wavelength infrared photodetectors // OptoElectr. Rev. 7, pp. 19-28 (1999).

17. J.J. Lee and M. Razeghi, Exploration of InSbBi for uncooled long-wavelength infrared photodetectors // Opto-Electr. Rev. 6, pp. 25-36 (1998).

18. W. E. Tennant, C. A. Cockrum, J. B. Gilpin, M. A. Kinch, M B. Reine and R. P. Ruth, Key issues in HgCdTe-based focal plane arrays: a industry perspective // J. Vac. Sci. Technol. B10, pp. 1359-369 (1992).

19. J. M. Arias, S. H. Shin, J. G. Pasko, R. E. DeWames, and E. R. Gertner, Long and middle wavelength infrared photodiodes fabricated with $\mathrm{Hg}_{1-\mathrm{x}} \mathrm{Cd}_{\mathrm{x}} \mathrm{Te}$ grown by molecular beam epitaxy // J. Appl. Phys. 65, pp. 1747-1753 (1989).

20. L. O. Bubulac, D. D. Edwall, D. McConnel, R. E. DeWames, E. R. Blazejewski, and E. R. Gertner, $p$-on- $n$ activated junction in LWIR HgCdTe/GaAs // Semicond. Sci. Technol. 5, 


\section{A. Rogalski: Heterostructure infrared photodiodes}

pp. S45-S48 (1990)

21. J. M. Arias, M. Zandian, J. G. Pasko, S. H. Shin, L. O. Bubulac, R. E. DeWames, and W. E. Tennant, Molecular-beam epitaxy growth and in situ arsenic doping of $p$-on- $n \mathrm{HgCdTe}$ heterojunctions // J. Appl. Phys. 69, pp. 2143-2148 (1991).

22. G. N. Pultz, P. W. Norton, E. E. Krueger, and M. B. Reine, Growth and characterization of $p$-on- $n$ HgCdTe liquid-phase epitaxy heterojunction material for 11-18 $\mu \mathrm{m}$ applications // J. Vac. Sci. Technol. B9, pp. 1724-1730 (1991).

23. C. C. Wang, Mercury cadmium telluride junctions grown by liquid phase epitaxy // J. Vac. Sci. Technol. B9, pp. 17401745 (1991).

24. M. B. Reine, K. R. Maschhoff, S. P. Tobin, P. W. Norton, J. A. Mroczkowski, and E. E. Krueger, The impact of characterization techniques on $\mathrm{HgCdTe}$ infrared detector technology // Semicon. Sci. Technol. 8, pp. 788-804 (1993).

25. P. R. Norton, Infrared image sensors // Opt. Eng. 30, pp. 1649-1663 (1991).

26. R. E. DeWames, J. M. Arias, L. J. Kozlowski and G. M Williams, An assessment of $\mathrm{HgCdTe}$ and GaAs/GaAlAs technologies for LWIR infrared imagers // Proc. SPIE 1735, pp. 2-16 (1992).

27. J. M. Arias, J. G. Pasko, M. Zandian, L. J. Kozlowski, and R. E. DeWames, Molecular beam epitaxy HgCdTe infrared photovoltaic detectors // Opt. Eng. 33, pp. 1422-1428 (1994).

28. M.B. Reine, E.E. Krueger, P. O'Dette, C.L. Terzis, B. Denley, J. Hartley, J. Rutter, and D.E. Kleinmann, Advances in $15 \mu \mathrm{m}$ $\mathrm{HgCdTe}$ photovoltaic and photoconductive detector technology for remote sensing // Proc. SPIE 2816, pp. 120-137 (1996).

29. S.P. Tobin, M.H. Weiler, M.A. Hutchins, T. Parodos, and P.W. Norton, Advances in composition control for $16 \mu \mathrm{m}$ LPE $p$-on- $n \mathrm{HgCdTe}$ heterojunction photodiodes for remote sensibg applications at $60 \mathrm{~K}$, to be published.

30. K. Vural, Mercury cadmium telluride short- and mediumwavelength infrared staring focal plane arrays // Opt. Eng. 26, pp. 201-208 (1987).

31. L. J. Kozlowski, R. B. Bailey, S. A. Cabelli, D. E. Cooper, I S. Gergis, A. C. Chen, W. V. McLevige, G. L. Bostrup, K. Vural, W. E. Tennant, and P. H. Howard, High-performance $5-\mu \mathrm{m} 640 \times 480 \mathrm{HgCdTe}$-on-sapphire focal plane arrays // Opt. Eng. 33, pp. 54-63 (1994).

32. T. Tung, L. V. DeArmond, R. F. Herald, P. E. Herning, M. H. Kalisher, D. A. Olson, R. F. Risser, A. P. Stevens, and S. J. Tighe, State of the art of Hg-melt LPE HgCdTe at Santa Barbara Research Center // Proc. SPIE 1735, pp. 109-134 (1992).

33. T.J. de Lyon, R.D. Rajavel, J.A. Vigil, J.E. Jensen, O. K Wu, C. A. Cockrum, S.M. Johnson, and G.M. Venzor, S.L. Bailey, I. Kasai, W.L. Ahlgren, and M.S. Smith, Molecularbeam epitaxial growth of $\mathrm{HgCdTe}$ infrared focal-plane arrays on silicon substrates for midwave infrared applications // J . Electron. Mater. 27, pp. 550-555 (1998).

34. L.J. Kozlowski, K. Vural, D.Q. Bui, R.B. Bailey, D.E. Cooper, and D.M. Stephenson, Status and direction of PACE-1 HgCdTe FPAs for astronomy // Proc. SPIE 1946, pp. 148-160 (1993).

35. L.J. Kozlowski, K. Vural, S.C. Cabelli, C.Y. Chen, D.E. Cooper, G.L. Bostrup, D.M. Stephenson, W.L. McLevige, R.B. Bailey, K. Hodapp, D. Hall, and W.E. Kleinhans, $2.5 \mu \mathrm{m}$ PACE-1 HgCdTe $1024 \times 1024$ FPA for infrared astronomy // Proc. SPIE 2268, pp. 353-364 (1994).

36. L.O. Bubulac, W.E. Tennant, J.G. Pasko, L.J. Kozlowski, M. Zandian, M.E. Motamedi, R.E. DeWames, J. Bajaj, N. Nayar,
W.V. McLevige, N.S. Gluck, R. Melendes, D.E. Cooper, D.D. Edwall, J.M. Arias, and R. Hall, High performance SWIR $\mathrm{HgCdTe}$ detector arrays // J. Electron. Mater. 26, pp. 649-655 (1997).

37. A. Rogalski and R. Ciupa, Performance limitation of short wavelength infrared InGaAs and $\mathrm{HgCdTe}$ photodiodes // to be published in J. Electron. Mater.

38. L.J. Kozlowski, K. Vural, J.M. Arias, W.E. Tennant, and R.E. DeWames, Performance of HgCdTe, InGaAs and quantum well GaAs/AlGaAs staring infrared focal plane arrays / / Proc. SPIE 3182, pp. 2-13 (1997).

39. G.H. Olsen and M.J. Cohen, Applications of near-infrared imaging // Proc. SPIE 3379, pp. 300-306 (1998).

40. A. Krier and Y. Mao, High performance uncooled InAsSbP/ InGaAs photodiodes for the 1.8-3.4 $\mu \mathrm{m}$ wavelength range // Infrared Phys. Technol. 38, pp. 397-403 (1997).

41. R.M. Lin, S.F. Tang, S.C. Lee, C.H. Kuan, G.S. Chen, T.P. Sun, and J.C. Wu, Room temperature unpassivated InAs $p-i-n$ photodetectors grown by molecular beam epitaxy // IEEE Trans. Electron Devices 44, pp. 209-213 (1997).

42. R.E. DeWames, D.D. Edwall, M. Zandian, L.O. Bubulac, J.G. Pasko, W.E. Tennant, J.M. Arias, and A. D'Souza, Dark current generating mechanisms in short wavelength infrared photovoltaic detectors // J. Electron. Mater. 27, pp. 722-726 (1998).

43. J. M. Arias, M. Zandian, G. M. Williams, E. R. Blazejewski, R. E. DeWames, and J. G. Pasko, HgCdTe dual-band infrared photodiodes grown by molecular beam epitaxy // J. Appl. Phys. 70, pp. 4620-4622 (1991).

44. E. R. Blazejewski, J. M. Arias, G. M. Williams, W. McLevige, M. Zandian, and J. Pasko, Bias-switchable dual-band HgCdTe infrared photodtetcor // J. Vac. Sci. Technol. B10, pp. 1626-1632 (1992).

45. M. B. Reine, P. W. Norton, R. Starr, M. H. Weiler, M. Kestigian, B. L. Musicant, P. Mitra, T. Schimert, F. C. Case, I. B. Bhat, H. Ehsani, and V. Rao, Independently accessed back-to-back $\mathrm{HgCdTe}$ photodiodes: A new dual-band infrared detector // J. Electr. Mater. 24, pp. 669-678 (1995).

46. R. D. Rajavel, D. M. Jamba, O. K. Wu, J. E. Jensen, J. A. Wilson, E. A. Patten, K. Kosai, P. Goetz, G. R. Chapman, and W. A. Radford, High performance $\mathrm{HgCdTe}$ two-color infrared detectors grown by molecular beam epitaxy // $J$. Crystal Growth 175, pp. 653-658 (1997).

47. R. D. Rajavel, D. M. Jamba, J. E. Jensen, O. K. Wu, C. Le Beau, J. A. Wilson, E. Patten, K. Kosai, J. Johnson, J. Rosbeck, P. Goetz, and S. M. Johnson, Molecular beam epitaxial growth and performance of integrated two-color $\mathrm{HgCdTe}$ detectors operating in the mid-wave infrared band // J. Electr. Mater. 26, pp. 476-481 (1997).

48. P. Mitra, S. L. Barnes, F. C. Case, M. B. Reine, P. O'Dette, R. Starr, A. Hairston, K. Kuhler, M. H. Weiler, and B. L. Musicant, MOCVD of bandgap-engineered HgCdTe $p-n-N-P$ dual-band infrared detector arrays // J. Electron. Mater. 26, pp. $482-487$ (1997).

49. M. B. Reine, A. Hairston, P. O’Dette, S. P. Tobin, F. T. J. Smith, B. L. Musicant, P. Mitra, and F. C. Case, Simultaneous MW/LW dual-band MOCVD HgCdTe 64×64 FPAs // Proc. SPIE 3379, pp. 200-212 (1998).

50. R.D. Rajavel, D.M. Jamba, J.E. Jensen, O.K. Wu, P.D. Brewer, J.A. Wilson, J.L. Johnson, E.A. Patten, K. Kasai, J.T. Caulfield, and P.M. Goetz, Molecular beam epitaxial growth and performance of $\mathrm{HgCdTe}$-based simultaneousmode two-color detectors // J. Electron. Mater. 27, pp. 747751 (1998) 